\title{
Methodik der Neurografie - motorische und sensible Neurografie
}

Christian Bischoff, Wilhelm Schulte-Mattler

\begin{abstract}
Die Messung der Amplitude und Nervenleitgeschwindigkeit dient der Bestimmung des Funktionszustandes peripherer Nerven. Im Speziellen untersucht die Neurografie: traumatische Nervenläsionen, Nervenkompressionssyndrome, Polyneuropathie (Beurteilung des pathophysiologischen Typs) sowie Plexopathie vs. Radikulopathie (sensible Neurografie).
\end{abstract}

\section{Allgemeine Anforderungen}

\section{Gerät und Raum}

- technische Prüfung des Geräts nach Einweisung

- Untersuchungsliege (fast alle Untersuchungen werden im Liegen durchgeführt)

- Raum störungsfrei (Lärm)

Vorbereitung und Ausführung Bereithalten von:

- Desinfektionsmittel

- Oberflächenelektroden (in unterschiedlichen Größen/ Längen) zur Ableitung

- Stimulationselektrode

- Elektrodengel

- Kabel mit passenden Anschlüssen

- Erdelektrode

- Reinigungsmaterial für Oberflächen (Liege)

- Tupfer ggf. zur Hautreinigung

- Anleitungsbuch (für selten untersuchte Nerven)

\section{Vorbereitung Patient}

- orientierende Untersuchung und Auswahl der Methode (motorisch/sensibel/F-Welle/welcher Nerv)

- Information über den Ablauf der Untersuchung

- Schrittmacher? (keine Stimulation in Schrittmachernähe)

- Lagerung auf Liege, auf entspannte Position achten

- Messung der Oberflächentemperatur am Ableitort

- ggf. Aufwärmen der Extremität (s.u.)

- ggf. Säubern der Ableitstelle

- Anbringen der Erdelektrode - auf guten Kontakt achten (ausreichend feucht bzw. ausreichende Kontaktpaste)

- bei starker Schweißbildung Trocknen der Haut

\section{Ausführung der Untersuchung}

- Eingabe der Patientendaten (ID, Name, Geburtsdatum, Untersucher)

- Auswahl des zu untersuchenden Nervs und der Untersuchungsmethode und Eingabe am Gerät (auf Seite achten)

- Lautsprecher aktivieren (Artefakterkennung!)

- Starten des Untersuchungsprogramms und Prüfen der Geräteeinstellungen

- Start der Untersuchung

- weiteres Vorgehen s. unten bei den einzelnen Modalitäten

- Bewertung der Messung - Fehler und Artefakte erkennen und beseitigen ( $\vee$ Tab. 1 )

- Speicherung und Dokumentation der Messergebnisse (digital; Papier)

- Entfernen von Elektroden, ggf. Elektrodengel abwischen

- Information an Patienten - Untersuchung beendet -

- Auswertung mit Bezug zum klinischen Befund

\section{Fehler und Artefakte erkennen und beseitigen}

Technische Fehler vermeiden: Checkliste prüfen

( $\triangleright$ Tab. 1)

\section{Biologische Artefakte}

- Unruhe bzw. Anspannen des Patienten - Lagerung des Patienten optimieren

- mangelnde Mitarbeit des Patienten - Information an Patienten wiederholen

- Temperatur an der Ableitstelle zu niedrig

- Befund normal $\rightarrow$ Ende der Untersuchung

- Befund pathologisch $\rightarrow$ Aufwärmen (z. B. Wasserbad; mind. 15 min; alternativ Wärmepads, bis Temperatur bei $\left.34^{\circ} \mathrm{C}\right) \rightarrow$ Wiederholung der Untersuchung 
\Tab. 1 Neurografie: mögliche Gerätefehler und Abhilfe.

\begin{tabular}{|l|l|}
\hline Gerätefehler & Abhilfe \\
\hline $\begin{array}{l}\text { Störung mit hoher Amplitude: } \\
\text { häufig defekte Kabelkontakte }\end{array}$ & Kabel wechseln - Elektrode wechseln \\
\hline „Brummen“ $=50 \mathrm{~Hz}$ & $\begin{array}{l}\text { - Erdung prüfen - Elektrodenübergangswiderstände prüfen } \\
\text { - lange Kabel miteinander verdrehen - abschirmen - kurze Kabel verwenden } \\
\text { - Störquellen abschalten (z. B. Leuchtstoffröhren etc.) }\end{array}$ \\
\hline kein Signal & $\begin{array}{l}\text { - Verstärker überprüfen (Eingang offen?) } \\
\text { - falsche Verstärkung }\end{array}$ \\
\hline Signal übersteuert & Verstärkung anpassen \\
\hline Signal nur akustisch & Zeitfenster anpassen \\
\hline keine Stimulation & Kabel überprüfen; Anfeuchten der Elektrode \\
\hline
\end{tabular}

\section{Technische Empfehlungen}

- Ableitung motorischer Antworten fast immer mit Oberflächenelektroden (Werte sonst nicht verwendbar; Nadelelektroden nur bei hochgradiger Atrophie zum Nachweis, dass der Nerv noch erregbar ist)

- Bei der sensiblen Neurografie sind Ableitungen mit Nadelelektroden möglich (Cave: andere Normalwerte)

- Stimulation: kurze Stimulationsdauer wählen (in der Regel 0,1-0,2 ms; wenn mit hohen Reizstärken eine nur submaximale Antwort ausgelöst wird, Verlängerung der Reizdauer)

- Tab. 2 fasst die technischen Empfehlungen zur Geräteeinstellung bei Ableitung zusammen.

\section{Auswertung und Normalwerte}

- Beschreibung des Untersuchungsergebnisses je nach Fragestellung (s.u.)

- Vergleich mit Normalwerten (je nach untersuchtem Nerv)

- Zusammenfassende Bewertung und Einordnung im klinischen Kontext

- schriftlicher Befund

- Tab. 2 Geräteeinstellung Neurografie.

\begin{tabular}{|l|l|}
\hline Verstärkung & $\begin{array}{l}0,2 \mathrm{mV} / \text { Div zur Latenzmessung } \\
\text { Anpassen zu Amplituden- } \\
\text { bestimmung }\end{array}$ \\
\hline Ablenkgeschwindigkeit & $2-5 \mathrm{~ms} / \mathrm{Div}$ \\
\hline Filter & $5 \mathrm{~Hz}-10 \mathrm{kHz}$ \\
\hline
\end{tabular}

\section{Spezielle Techniken}

\section{Motorische Neurografie}

\section{Technische Besonderheiten}

Messung der Nervenleitgeschwindigkeit nur bei Stimulation an mind. 2 Punkten möglich (Latenz der neuromuskulären Transmission an der Endplatte geht in die Latenz mit ein und ist nicht bestimmbar; $\triangleright$ Abb. 1).

\section{Ausführung der Untersuchung}

- erste Schritte siehe allgemeiner NLG-Methoden-Teil

- Inspektion der Ableitstelle (Verschmutzung, Schweiß), ggf. reinigen

- Aufkleben der Elektroden in „Belly-Tendon“-Technik (Ableitelektrode über dem Muskelbauch; Referenzelektrode über elektrisch „inaktiver“ Stelle (Sehne, Knochen)

- Abmessung der normierten (vorgegebenen) Distanz zwischen Stimulations- und Ableitelektrode

- Suche der Stimulationsstelle (nervennah) unter der Kathode zunächst mit niedriger Stimulationsintensität

- immer Beobachtung des Stimulationserfolges (Kontraktion des Zielmuskels)

- nach Auffinden der optimalen Stimulationsstelle Steigerung der Stimulationsintensität bis zur Erreichung der supramaximalen Stimulation (kein weiterer Anstieg der Amplitude)

- supramaximale Stimulation an einer proximalen Stelle

- bei N. ulnaris und N. peronaeus fraktionierte Neurografie (Distanz möglichst $10 \mathrm{~cm}$ ) oder Inching (Stimulation entlang des Nervs in kurzen Abständen (1 oder $2 \mathrm{~cm}$ ), um die Schädigungsstelle zu lokalisieren)

- Streckenmessung und Eingabe der Werte 


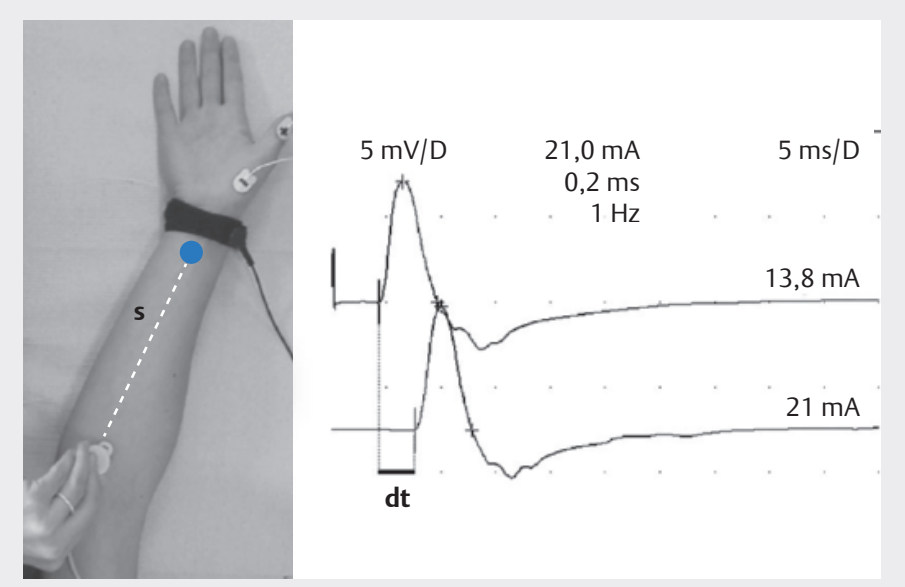

- Abb. 1 Motorische Neurografie. Prinzip der motorischen Neurografie am Beispiel des $\mathrm{N}$. medianus mit Stimulation am Handgelenk und in der Ellenbeuge sowie Ableitung des MSAP vom M. abductor pollicis brevis. Quelle: aus Bischoff et al. EMG NLG. Thieme, 2014.

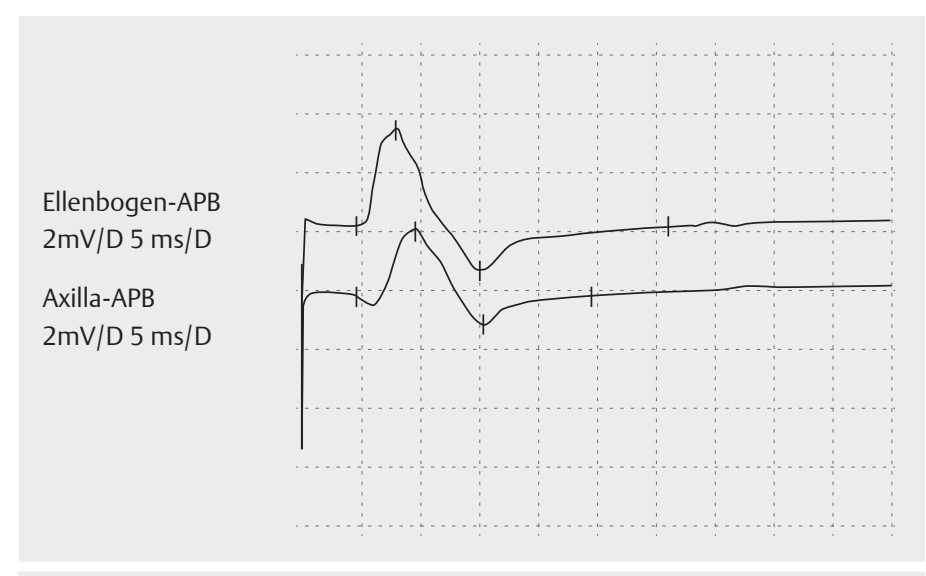

- Abb. 2 Bestimmung der DML. Der Latenzmarker wird am Abgang des Potenzials von der Grundlinie (nach positiv oder negativ) gesetzt. Obere Spur: korrekte Elektrodenposition im Endplattengebiet (Motor Point; negativer Abgang). Untere Spur: Elektrodenposition außerhalb der Endplattenregion mit positiver Vorwelle.

\section{Messwerte}

- DML (distal motorische Latenz): Latenz zwischen dem Stimulus und dem Potenzialbeginn (Messung bis zur ersten Abweichung von der Grundlinie, unabhängig davon ob nach negativ oder positiv) ( $\bullet$ Abb. 2) immer bei normierter Distanz

- Amplitude proximal und distal (bei den Werten beachten, ob die Normalwerte Peak-Peak oder BaselinePeak bestimmt wurden)

- Konfiguration der MSAP und Konfigurationsänderung an unterschiedlichen Stellen

- Nervenleitgeschwindigkeit: v=(Streckendifferenz zwischen der Kathodenposition bei distaler und proximaler Stimulation) $/(\mathrm{s} / \mathrm{dt}=$ Latenzdifferenz zwischen distaler und proximaler Stimulation) ( $\bullet$ Abb. 1)

\section{Cave}

Fallen/Probleme

- NLG-Wert repräsentiert den Wert nur der schnellstleitenden Nervenfaser im untersuchten Abschnitt

- Längenmessfehler bei Stimulationen über Gelenke, daher möglichst immer in der gleichen Winkelstellung untersuchen

- Proximale niedrige Amplitude:

- submaximale Stimulation an proximalen Stellen (bei tiefliegenden Nerven)

- Leitungsblock

- Anastomose (z. B. Martin-Gruber-Anastomose Fasern des N. ulnaris laufen am Ellbogen zusammen mit dem N. medianus)

- Konfigurationsänderung der MSAP

- Mitstimulation eines benachbarten Nervs

- temporale Dispersion bei Demyelinisierung

- Markersetzung bei geringer Verstärkung $\rightarrow$ Latenz fälschlich zu lang bestimmt

- bei langer Latenz (Demyelinisierung) Anpassung der Ablenkgeschwindigkeit (Dauer der Darstellung)

- bei Muskelatrophie kann Elektrodenposition falsch gewählt werden

- bei hoher Stimulationsintensität (Ko-)Aktivierung benachbarter Nerven

- keine Verwendung von Blockelektroden

\section{Referenzwerte der motorischen Neurografie} Siehe $>$ Tab. 3. 
- Tab. 3 Referenzwerte der motorischen Neurografie (Werte nicht größen- und alterskorrigiert; Ableittemperatur mind. $32^{\circ} \mathrm{C}$; n. b.: nicht bestimmbar).

\begin{tabular}{|c|c|c|c|c|}
\hline & $\begin{array}{l}\text { DML } \\
\text { Ableitdistanz } \\
{[\mathrm{cm}]}\end{array}$ & $\begin{array}{l}\text { DML } \\
\text { obere Normgrenze } \\
\text { [ms] }\end{array}$ & $\begin{array}{l}\text { MSAP-Amplitude } \\
\text { untere Normgrenze } \\
{[\mathrm{mV}]}\end{array}$ & $\begin{array}{l}\text { NLG } \\
\text { untere Normgrenze } \\
{[\mathrm{m} / \mathrm{s}]}\end{array}$ \\
\hline \multicolumn{5}{|l|}{ Schulter-Arm-Nerven } \\
\hline N. axillaris & $14-20$ & 5,0 & 10 & n.b. \\
\hline N. thoracicus longus & $20-25$ & 5,3 & 2,5 & n.b. \\
\hline N. musculocutaneus & 25 & 5,9 & 5 & n.b. \\
\hline N. medianus & 7 & 4,2 & 8 & $\begin{array}{l}\text { Unterarm } 48 \\
\text { proximal } 55\end{array}$ \\
\hline N. ulnaris & 7 & 3,5 & 8 & $\begin{array}{l}\text { Unterarm } 50 \\
\text { proximal } 55\end{array}$ \\
\hline N. radialis & 10 & 3,4 & 8 & 50 \\
\hline \multicolumn{5}{|l|}{ Beinnerven } \\
\hline N. femoralis & 16 & 5,6 & 4 & n.b. \\
\hline N. peronaeus & 8 & 5,6 & 5 & 42 \\
\hline N. tibialis & $8-10$ & 6,0 & 8 & 40 \\
\hline \multicolumn{5}{|l|}{ Gesichtsnerven } \\
\hline N. facialis & 5 & 4,1 & & \\
\hline
\end{tabular}

\section{F-Welle}

\section{Besonderheiten}

- motorische Spätantwort durch retrograde Erregungsleitung mit synapsenfreier Spiegelung am Alpha-Motoneuron

- Amplitude 1-5\% der M-Antwort

Ausführung der Untersuchung Erste Schritte siehe allgemeiner NLG-Methoden-Teil.

- Inspektion der Ableitstelle (Verschmutzung, Schweiß), ggf. reinigen

- Aufkleben der Elektroden in „Belly-Tendon“-Technik (Ableitelektrode über dem Muskelbauch; Referenzelektrode über „elektrisch inaktiver Stelle“ (Sehne, Knochen)

- Suche der Stimulationsstelle (nervennah) der Kathode, zunächst mit niedriger Stimulationsintensität; Kathode möglichst nach proximal ausgerichtet

- nach Auffinden der optimalen Stimulationsstelle Steigerung der Stimulationsintensität bis zur Erreichung der supramaximalen Stimulation (Kennzeichen: kein weiterer Anstieg der Amplitude)

- 10 bzw. 20 supramaximale Stimulationen an derselben Stelle, möglichst in unregelmäßigen Abständen

- auf Entspannung des Patienten achten (Muskelartefakte)

\section{Messwerte}

- kürzeste F-Wellen Latenz oder kürzeste F-M-Latenz (bezogen auf die Körperlänge)

- Persistenz (Zahl der auslösbaren F-Wellen)

- Chronodispersion und Amplitude weniger bedeutend

- Referenzwerte der F-Welle sind in > Tab. $\mathbf{4}$ aufgelistet.

Fallen/Probleme

- auch bei nur geringer Willkürinnervation (akustische Kontrolle) Latenzbestimmung unmöglich (Muskelartefakte)

- bei niedrigen Amplituden ( $<1 \mathrm{mV}$ ) oft keine F-Welle abgrenzbar

- große Variabilität der Persistenz und Konfiguration ( $>$ Abb. 3)

- bei schneller Repetitionsfrequenz Habituation möglich

- Verwechslungsgefahr mit A-Wellen (konstante Latenz, Amplitude und Konfiguration) (> Abb. 3)

- Schädigungsort nicht eindeutig bestimmbar

- bei langer Latenz Anpassung der Ablenkgeschwindigkeit

- gelegentlich auch bei Gesunden keine F-Wellen des N. peroneus 
- Tab. 4 Referenzwerte der F-Wellen. Angegeben sind die Obergrenzen für die minimale Latenz, die Seitendifferenz, die Chronodispersion sowie die minimale Zahl der auslösbaren Antworten. Ableitbedingung: supramaximale Stimulation, entspannter Patient, 20 Stimuli, Oberflächenableitung. Diese Werte stellen nur grobe Anhaltszahlen dar, da weder das Alter noch die Körpergröße bzw. die Extremitätenlänge berücksichtigt sind.

\begin{tabular}{|l|l|l|l|l|l|}
\hline Nerv & Stimulationsort & $\begin{array}{l}\text { minimale } \\
\text { F-Latenz: oberer } \\
\text { Grenzwert [ms] }\end{array}$ & $\begin{array}{l}\text { maximale Seiten- } \\
\text { differenz der F-Latenz } \\
\text { [ms] }\end{array}$ & $\begin{array}{l}\text { Chronodispersion: } \\
\text { oberer Grenzwert [ms] }\end{array}$ & $\begin{array}{l}\text { Auslösbarkeit } \\
\text { (> n/20 Stimuli) }\end{array}$ \\
\hline N. medianus & Handgelenk & 30,8 & 2,5 & 13,2 & $12 / 20$ \\
\hline N. ulnaris & Handgelenk & 29,8 & 2,5 & 10,8 & $11 / 20$ \\
\hline N. peronaeus & Sprunggelenk & 53,1 & 4,2 & 15,8 & $12 / 20$ \\
\hline N. tibialis & Sprunggelenk & 58,0 & 4,5 & 19,5 & $16 / 20$ \\
\hline Bischoff C, Dengler R, Hopf HC. EMG NLG. Stuttgart: Thieme Verlag; 2014 & & \\
\end{tabular}

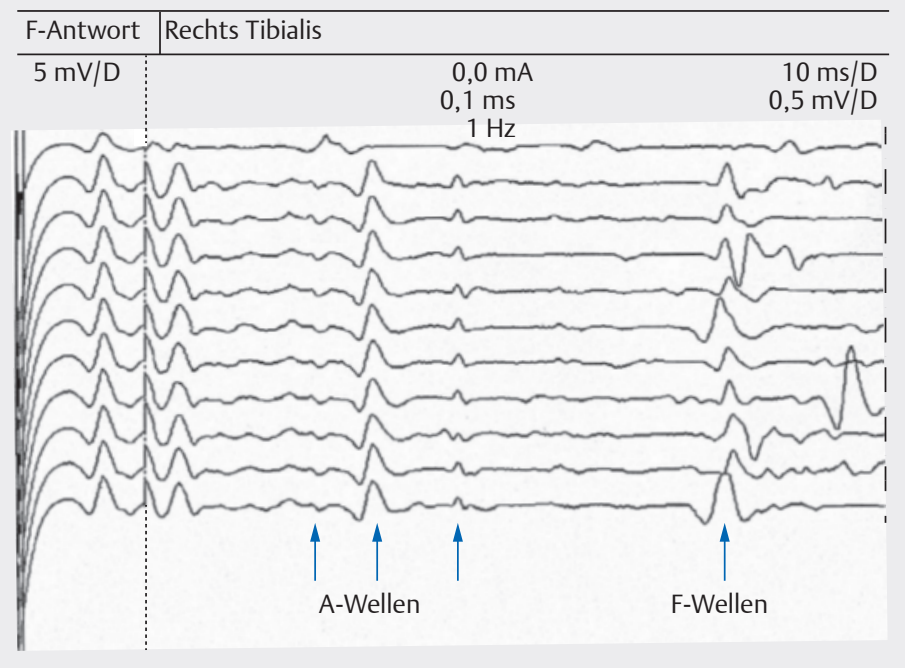

- Abb. 3 F-Wellen mit mehreren vorausgehenden A-Wellen.

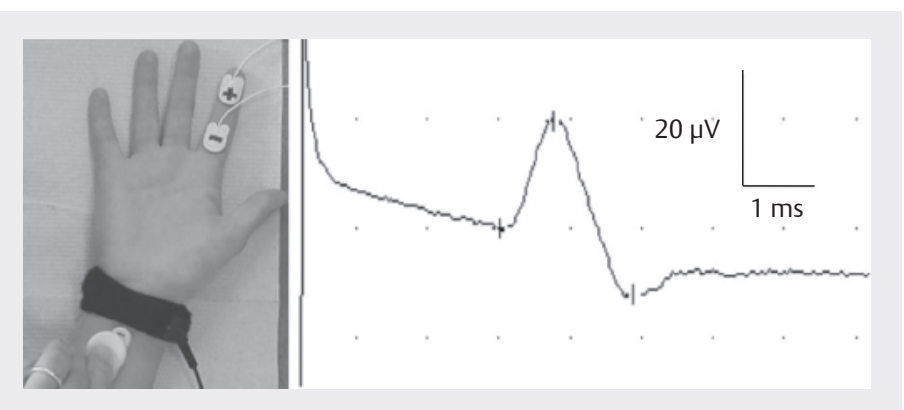

Abb. 4 Sensible Neurografie. Prinzip der sensiblen Neurografie mit antidromer Stimulation des N. medianus und einem SNAP.

Quelle: nach Bischoff et al. EMG NLG. Thieme, 2014

\section{Sensible Neurografie ( $\bullet$ Abb. 4)}

\section{Technische Besonderheiten}

- NLG-Bestimmung zwischen Stimulations- und Ableitort möglich

- niedrige Amplitude daher artefaktanfällig (Erdelektrode zwischen Stimulation und Ableitung)

- Untersuchung anti- und orthodrom möglich

Ausführung der Untersuchung Erste Schritte siehe allgemeiner NLG-Methoden-Teil.

- Inspektion und ggf. Reinigung der Haut bei Verschmutzung, Schweißbildung, vermehrter Hornhaut

- Aufkleben der Elektroden (anti- oder orthodrom)

- Suche der Stimulationsstelle (bei antidromer Technik nervennah) zunächst mit niedriger Stimulationsintensität; Frage an den Patienten, wo er es wahrnimmt

- nach Auffinden der optimalen Stimulationsstelle Steigerung der Stimulationsintensität bis zur Erreichung der supramaximalen Stimulation (kein weiterer Anstieg der Amplitude); Cave: motorisches Artefakt

- Streckenmessung und Eingabe der Werte

\section{Messwerte}

- Nervenleitgeschwindigkeit (möglichst bei normierter Distanz)

- Amplitude und Konfiguration des SNAP 
- Tab. 5 Referenzwerte der sensiblen Neurografie (Ableittemperatur $>32^{\circ} \mathrm{C}$ ).

\begin{tabular}{|l|l|l|l|}
\hline & $\begin{array}{l}\text { Ableitdistanz } \\
{[\mathbf{c m}]}\end{array}$ & $\begin{array}{l}\text { NLG }[\mathbf{m} / \mathbf{s}] \\
\text { untere Normgrenze }\end{array}$ & $\begin{array}{l}\text { SNAP-Amplitude }[\mu \mathrm{V}] \\
\text { untere Normgrenze }\end{array}$ \\
\hline Armnerven & & & 12 \\
\hline N. medianus & 7 & 45 & 15 \\
\hline N. ulnaris & 7 & 50 & 16 \\
\hline R. superficialis n. radialis & 10 & 55 & 12 \\
\hline N. cutaneus antebrachii & 12 & 57 & \\
\hline Beinnerven & & & 4 \\
\hline N. cutaneus femoris lateralis & $17-20$ & 43 & 5 \\
\hline N. saphenus & 15 & 46 & 10 \\
\hline N. peronaeus superficialis & $12-15$ & 40 & 10 \\
\hline N. suralis & 14 & 42 & \\
\hline
\end{tabular}

\section{Cave}

Fallen/Probleme

- Die NLG gibt nur den Wert der schnellstleitenden Nervenfaser in dem untersuchten Abschnitt wieder.

- Mit zunehmender Distanz zwischen Stimulationsund Ableitort nimmt die Amplitude des SNAP ab.

- bei präganglionären Schädigungen bleiben SNAP auslösbar

- bei großem Stimulationsartefakt Problem der Latenzbestimmung

- bei gleichzeitiger motorischer Antwort (oft bei antidromer Stimulation) Amplitudenbestimmung mitunter nicht möglich

- bei Mittelwertbildung unter Umständen Artefakte $\rightarrow$ bei Mittelwertbildung Antwort mind. 1-mal reproduzieren

- Markersetzung bei geringer Verstärkung $\rightarrow$ Latenz fälschlich zu lang bestimmt

- zur Ableitung Verwendung von Blockelektroden vorteilhaft

- bei antidromer Stimulation mitunter störende motorische Artefakte, bei orthodromer Stimulation niedrige Amplituden (dabei Verwendung von Nadelelektroden oft besser)

\section{Referenzwerte der sensiblen Neurografie}

Siehe $>$ Tab. 5.

\section{Befunde}

\section{Leitungsblock}

- Amplitudenabnahme bei gleicher Konfiguration oder -verlust ohne Änderung von Form oder Dauer der MSAP, bei supramaximaler Stimulation proximal der

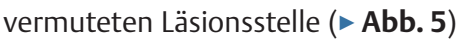

- erniedrigte Persistenz oder Ausfall der F-Wellen

\section{Pave}

- erst nach Abschluss der Waller'schen Degeneration (etwa 7-10 Tage) sicher

- Verwechslungsgefahr

- bei submaximaler Stimulation (bes. bei tiefer liegenden Nerven)

- bei Anastomosen

- Bei Leitungsblöcken, die distal der distalen Stimulationsstelle sind, kann in der Regel kein Amplitudensprung erfasst werden (z. B. Handgelenk).

- Bei der sensiblen Neurografie ist eine Amplitudenabnahme streckenabhängig physiologisch. 


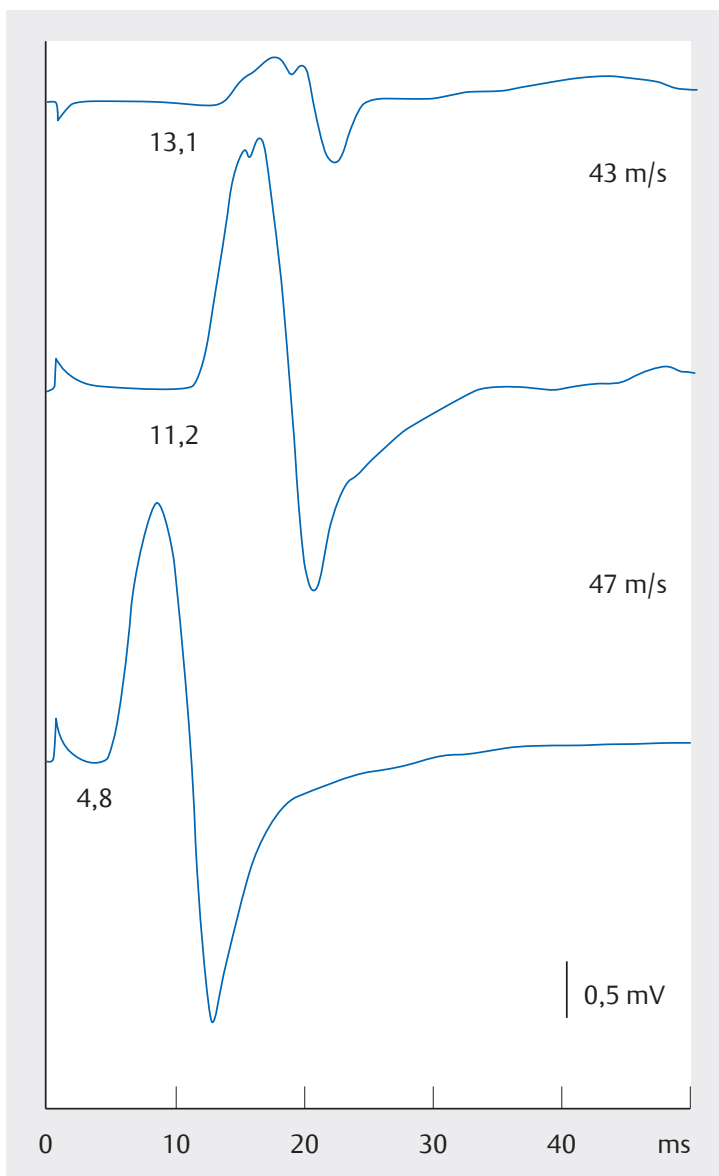

Abb. 5 Leitungsblock bei der proximalen Stimulation (oberste Spur). Abnahme der Amplitude (hier um 75\%) ohne Änderung der Dauer und Form.

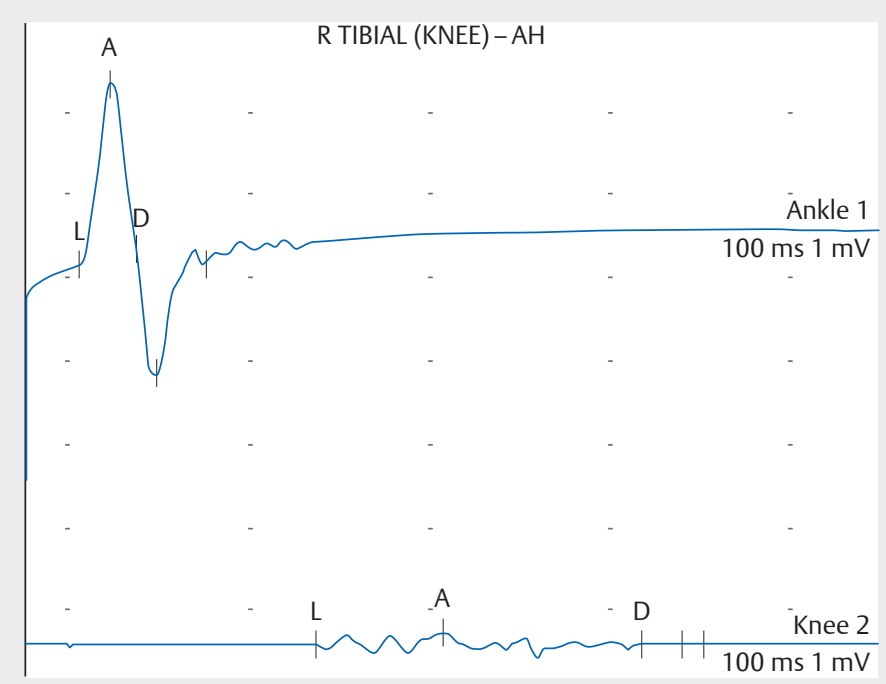

- Abb. 6 CIDP bei ausgeprägter Demyelinisierung. Bei proximaler Stimulation aufgesplittertes und in der Amplitude erniedrigtes Potenzial durch die temporale Dispersion bei ausgeprägter Demyelinisierung im Rahmen einer CIDP.

\section{Demyelinisierung}

- Abnahme der Nervenleitgeschwindigkeit im betroffenen Segment > 30\% des unteren Normwertes

- Aufsplitterung und ggf. Amplitudenabnahme des Potenzials infolge der temporalen Dispersion bei erworbenen Neuropathien ( $\bullet$ Abb. $\mathbf{6}$ ), in der Regel nicht bei hereditären Neuropathien

- pathologisch verlängerte Latenz der F-Wellen (>30\% des oberen Grenzwerts)

- pathologische DML (> 50\% des oberen Normwertes)

Cave

Fallen/Probleme

- auch bei axonalen Schädigungen niedrige NLG möglich (bis $30 \%$ des unteren Grenzwerts)

- niedrigere Temperatur in Nervennähe, NLG kann niedrig sein

- Streckenmessfehler

- bei erworbenen Neuropathien unterschiedliche NLG in unterschiedlichen Segmenten

\section{Axonale Läsion}

- Amplitudenabnahme oder Amplitudenverlust an allen Stimulationsstellen

- Erniedrigung der NLG bis 70\% des unteren Normwertes

- pathologische EMG-Befunde (siehe $>$ Tab. 4)

Cave

Fallen/Probleme

- Lokalisation des Schädigungsortes damit nicht möglich

- erst nach 7 Tagen erkennbar (Waller'sche Degeneration)

- Ursachen niedriger Amplituden s. o.

\section{Befundinterpretation}

\section{Überall kleine MSAP-Amplitude}

- axonale Läsion

- submaximale Stimulation

- Myopathie

- Demyelinisierung (temporale Dispersion/Aufsplitterung)

- alle Stimulationen proximal eines (distalen)Leitungsblocks

- LEMS

\section{Proximales MSAP kleiner als distales}

- Leitungsblock ( $\triangleright$ Abb. 5)

- temporale Dispersion (Aufsplitterung, Konfigurationsänderung) (॰ Abb. 6)

- submaximale Stimulation

- Anastomose 


\section{Kleine SNAP-Amplitude}

- axonale Läsion

- submaximale Stimulation

- Demyelinisierung (temporale Dispersion/Aufsplitterung), dabei auch fehlendes SNAP

\section{Niedrige NLG}

- Demyelinisierung (N. medianus < $38 \mathrm{~m} / \mathrm{s}$; Bein < $30 \mathrm{~m} /$ s)

- axonaler Schaden (bis 70\% des unteren Grenzwertes)

- niedrige Temperatur

\section{Repetitive (Serien-)Stimulation}

Definition Methode zur Beurteilung der Funktion der neuromuskulären Übertragung.

Ausführung der Untersuchung Erste Schritte siehe allgemeiner NLG-Methoden-Teil.

- Aufkleben der Oberflächenelektroden in „Belly-Tendon“-Technik (Ableitelektrode über dem Muskelbauch; Referenzelektrode über „elektrisch inaktiver Stelle“ (Sehne, Knochen)

- typische Ableite-/Stimulationsorte:

- N. facialis/M. mentalis, M. nasalis oder M. orbicularis oculi

- N. accessoris/M. trapezius

- N. axillaris/M. deltoideus

- Suche der Stimulationsstelle

- nach Auffinden der optimalen Stimulationsstelle Steigerung der Stimulationsintensität bis zur Erreichung der supramaximalen Stimulation (Kennzeichen: kein weiterer Anstieg der Amplitude)

- Fixation der Stimulationselektrode

- Verhinderung von Muskelbewegungen (wenn möglich)

- Durchführung der Serienreizung: 10 Stimuli mit 3/s

- auf Entspannung des Patienten achten (Muskelartefakte)

- ggf. Wiederholung 5 min nach einer tonischen Innervation für $30 \mathrm{~s}$

- bei V.a. Lambert-Eaton-Syndrom: Stimulation in Ruhe und unmittelbar nach einer tonischen Innervation für $5-10 s$

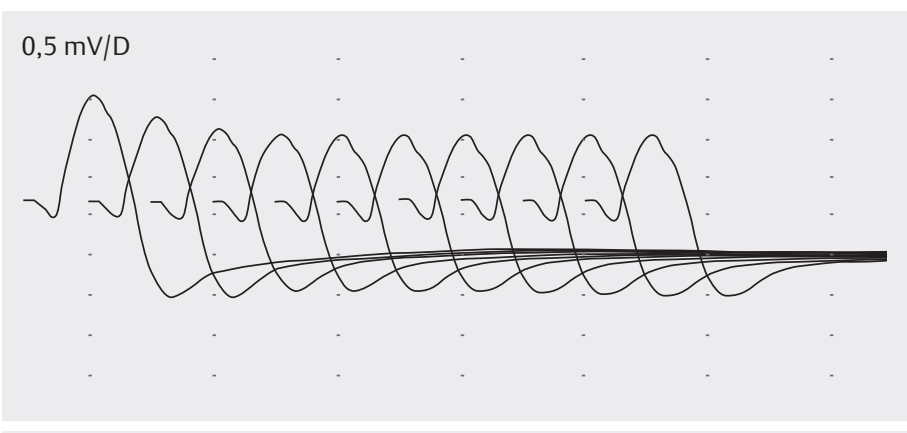

- Abb. 7 3/s Serienstimulation mit einem pathologischen Dekrement bei einem Patienten mit Myasthenia gravis.

\section{Messwerte}

- 3-Hz-Stimulation: Vergleich der Amplitude des 4. oder 5. Potenzials mit dem 1.; pathologisch ist ein reproduzierbares Dekrement $>10 \%$ ( $>$ Abb. 7)

- LEMS: Vergleich der Amplitude vor und nach tonischer Innervation; pathologisch ist eine Amplitudenzunahme $>50 \%$

Cave

Fallen/Probleme

- Bei submaximaler Stimulation kann es zu einer Pseudofazilitierung kommen.

- Bei der Seriensimulation kann es durch Muskelbewegung zu Änderungen der Konfiguration kommen.

- Bei der Myasthenia gravis bleibt die Amplitude nach dem 5. Stimulus konstant oder nimmt wieder gering zu.

- Ein Dekrement kann fälschlicherweise nicht nachweisbar sein:

- bei niedriger Muskeltemperatur

- unmittelbar nach einer Kraftprüfung

- bei Therapie mit einem Cholinesterasehemmer

- Ein pathologisches Dekrement kommt vor bei

- Myasthenie

- Lambert-Eaton-Myasthenie-Syndrom

- Reinnervationsprozessen

- Besonders bei okulären Myasthenien kann ein Dekrement fehlen. 
Interessenkonflikt

Die Autoren geben an, dass kein Interessenkonflikt besteht.

Autorinnen/Autor

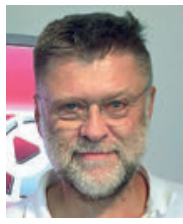

\section{Wilhelm Schulte-Mattler}

Prof. Dr. med. Wilhelm Schulte-Mattler schloss nach einem Studium der Mathematik und Physik sein Medizinstudium 1988 an der Universität Würzburg ab, wurde dort 1991 über „Die klinische Standard-Elektromyographie: Neue Möglichkeiten der Quantifizierung“ promoviert. Facharzt für Neurologie 1993. Oberärztliche Leitung der EMG-Labors der Neurologischen Universitätskliniken Halle (Saale) 1994 bis 2000, Regensburg 2000 bis 2019. Habilitation 2002, Professor seit 2009.

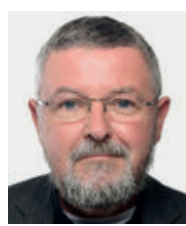

\section{Christian Bischoff}

Prof. Dr. med. 1979-1984 Studium in Marburg/ Lahn. Weiterbildung zum Neurologen Städtische Kliniken Kassel, Universität Göttingen und TU München. 1993 Fellow Dept. of Clinical Neurophysiology, University Uppsala, Schweden. 1992-1997 Oberarzt, 1997-2002 Leitender Oberarzt und Stellvertreter des Klinikdirektors der Neurologischen Klinik der TU München. 1994 Habilitation. 2001 apl. Professor für Neurologie. Seit 2002 Niederlassung in der Neurologischen Gemeinschaftspraxis am Marienplatz, München. Schwerpunkte: klinische Neurophysiologie, EMG, neuromuskuläre Erkrankungen.

Korrespondenzadresse

Prof. Dr. med. Wilhelm Schulte-Mattler

Klinik und Poliklinik für Psychiatrie und Psychotherapie der Universität Regensburg

Universitätsstraße 84

93053 Regensburg

wilhelm.schulte-mattler@klinik.uni-regensburg.de

\section{Erstveröffentlichung}

Dieser Beitrag wurde veröffentlicht in: Bischoff C, Buchner H Hrsg. SOPs Klinische Neurophysiologie, 1. Auflage. Stuttgart: Thieme; 2018.

Literatur

[1] Bischoff C, Schulte-Mattler W. Das EMG Buch. 4. Aufl. Stuttgart: Thieme; 2016

[2] Bischoff C, Dengler R, Hopf H-C. EMG - NLG. 3. Aufl. Stuttgart: Thieme; 2014

[3] Kimura J. Electrodiagnosis in Diseases of Nerve and Muscle. 4th ed. Oxford: Oxford University Press; 2013

[4] Preston DC, Shapiro BE. Electromyography and neuromuscular Disorders. Boston: Butterworth Heinemann; 1998

[5] Stöhr M, Pfister R. Klinische Elektromyographie und Neurographie. 6. Aufl. Stuttgart: Kohlhammer; 2014

\section{Bibliografie}

DOI https://doi.org/10.1055/a-0881-6592

Neurologie up2date 2019; 2: 115-124

(c) Georg Thieme Verlag KG Stuttgart · New York ISSN 2511-3453 